Journal of Computer Science 3 (7): 461-464, 2007

ISSN 1549-3636

(C) 2007 Science Publications

\title{
SSM: Slot Scheduling Mechanisms in a Multihop Wireless Hotspot Network
}

\author{
Maan Younis Abdullah \\ College of Computer Sciences and Mathematics, \\ Mousl University, Iraq
}

\begin{abstract}
Our focus was to determine a "slot" time for message forwarding. To this end, we model the system as a "Queue" where the time for packet forwarding is determined by slot, at different slot range for each relaying node. We also introduced one important reason of the poor performance is the close coupling between medium contention and network congestion. Our simulation results revealed how that the slot scheduling mechanisms are able to guide the multihop wireless network into an equilibrium state quickly. We also showed that maintaining communication among the relaying nodes was important for a stable MWN.
\end{abstract}

Key words: Mobile ad hoc networks, hotspot, scheduling

\section{INTRODUCTION}

In the Ad Hoc network, mobile nodes move constantly so that the topology of the network is dynamic, the links between nodes are unreliable, even broken, etc,. These uncertainties are related to the geographical locations. Likewise, when a mobile node has already had lots of data to process, there would be a large delay for the data that arrive later to be able to get processed. If there is data continuously that arrive at this node, there is no doubt that there would be a congestion. We call this uncertainties related to information transmission. When there is link broken or congestion, the data that have to pass this node will have a big delay, or even in worse cases, get lost. So it is needed to get an alternative route to avoid the congested area. This is called as route maintenance that are methods or strategies used to find a route with the least overhead to the designated destination node under dynamic circumstances with uncertainties. It's also called as route planning.

Existing fair scheduling mechanisms in ad hoc networks can be classified into two categories: timestamp based ${ }^{[1]},{ }^{[2]}$ and credit based ${ }^{[3]}$, according to different decision metrics. The timestamp-based mechanisms in ${ }^{[1]}$ and ${ }^{[2]}$ work similarly. In ${ }^{[2]}$, a flow graph must first be generated. Each vertex in the flow graph represents a flow. When two flows contend for the same resource, an edge is added between them. The scheduling discipline in ${ }^{[2]}$ is based on start-time fair queuing (SFQ) ${ }^{[4]}$

In ${ }^{[3]}$, a two-tier and cluster-based mechanism is proposed. The network is logically partitioned into clusters, each with a scheduler. Each flow is associated with three parameters: credit, usage, and excess, where Excess Usage Credit. The scheduler assigns time slots to mobiles in the respective cluster based on the first tier algorithm. The mobiles scheduled to send at the next time slot then in turn assign the time slot to the flows determined by the second tier algorithm. Each cluster scheduler operates independently. The scheduling disciplines in both algorithms are all based on the credit value, i.e., the one with the smallest excess value has the priority to transmit. The unused credit can be accumulated for future use.

In this paper, we focus on providing incentive for message forwarding in a two-hop hotspot network. Since message forwarding consumes a RN's resources such as bandwidth and energy, a selfish RN would not be willing to forward other's message without an incentive mechanism. In this paper, we adopt the "take site" incentive model, i.e., the clients wait the RNs to forward their messages in queue with specific waiting slot.

Our focus in this paper is to determine a "fair" slot for the message forwarding service in this network. To this end, we model the system as a Queue where the Slot for message forwarding is determined by Queue length.

The rest of the paper is organized as follows. In Section 2, we give some background and related works 
in packet scheduling schemes in ad hoc networks. In Section 3 we discuss in detail the proposed SSM Algorithm. This is followed by simulation result in Section 4, and conclude the paper in Section 5.

To avoid congestion and bottlenecks in ad hoc networks, traditional techniques such as load balancing and multi-path routing are normally adopted. These techniques used end-to-end approach in resolving congestion issues. For load balancing schemes ${ }^{[5,6,7]}$, route requests RREQ sent by source nodes gather load or quality of path information as they travel towards the destination. Amongst the several routes discovered, the destination or the source chooses the least loaded path for data transfer Multi-path routing ${ }^{[7,8]}$ on the other hand establishes multiple paths between.

In order to connect to the base station, the faraway nodes may rely on the intermediate nodes to forward their packets. This is the multihop wireless hotspot network proposed in recent literatures ${ }^{[9,10]}$. It is a hybrid network consisting of infrastructure and ad-hoc networking components. We consider a multihop hotspot network as illustrated in Figure 1. In this architecture, a mobile client may not be able to reach the base station (BS) via single-hop direct communication. Instead, the client must rely on another node who is closer to the BS to forward its packets. Such nodes are called the relaying nodes (RN).

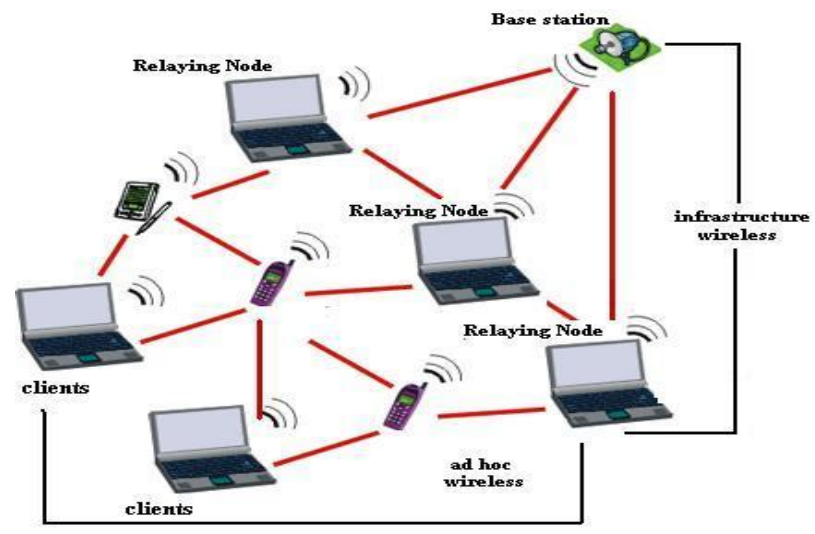

Fig. 1: Multihop Wireless Hotspot Network

Different packet scheduling schemes have been employed in conventional wired networks. It has been proven in ${ }^{[11]}$ that a scheme that schedules the small- est packet first results in the least end-to-end packet de- lay. This idea is analogous to scheduling the shortest job first on a multi-programmed processor. In infrastructured wireless networks, scheduling has been used to implement fairness of resource allocation among various subscribers being serviced by a base station. A solution to this problem has been proposed in ${ }^{[12]}$. A similar problem pertaining to ad hoc networks has been addressed in ${ }^{[13]}$.
In this paper, the movement of mobile nodes in environments with attraction places and routes are intensively studied. By using the approach introduced in ${ }^{[14]}$, the Hotspot model and the Route model are proposed and simulated.

\section{Proposed Algorithm}

Slot Curve: The waiting of the message $(\mathrm{m})$ is related to its sum queuing slot .

$$
S_{m}=\sum_{1}^{k} q_{k}+m
$$

where $S_{m}$ is the maximum time for sending message $(m), m$ is the current message time requirement,$q_{k}$ is the time require for each message, and $k$ is the No of message inside waiting queue.

$$
S=\frac{1}{S_{m}+Q} .
$$

When the, quantity is high, the slot is low. This relation can be captured by a function called slot curve. Fig. 2 . It has the following mathematical definition

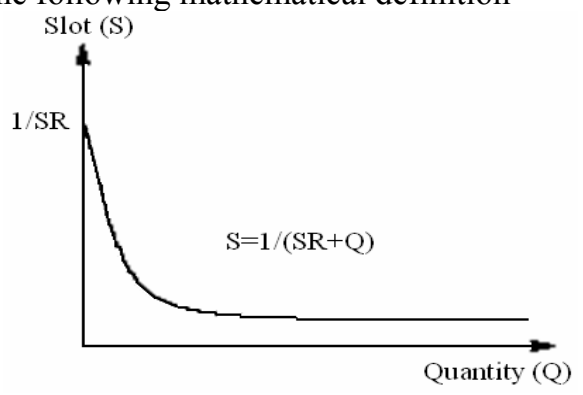

Fig. 2: Quantity curve

where $S$ is the slot, $Q$ is the quantity of messages. The $\mathrm{Q}$ is measured by the amount of traffic a client is requesting messages. When the slot time is low, the client message more traffic.

In each node is ranked with a slots based on its messages forwarding behavior observed by other nodes in the same neighborhood. A node with high slot is then reduce service by other nodes from the network. If the slot time of a current message is prohibitively high.

SSM algorithm: The general system algorithm of SSM is described as follows:

1. A multihop wireless network is considered in which a TDMA-based system is assumed to operate over a single channel shared by all hosts. The bandwidth is represented as frames of time slots and the slot allocation to messages waiting to be transmitted is based on their service tags. Each TDMA frame contains a fixed number of time slots. The network is synchronized on a frame and slot basis, using existing synchronization protocols $[15,16]$ 
Table 2: Result of optimal slot (S)

\begin{tabular}{|c|c|c|c|c|c|c|c|}
\hline \multirow{2}{*}{ M } & \multirow{2}{*}{$\begin{array}{l}\text { Start } \\
\text { node }\end{array}$} & \multirow{2}{*}{$\begin{array}{l}\text { End } \\
\text { node }\end{array}$} & \multirow{2}{*}{ Route } & \multicolumn{3}{|c|}{ Single-hop message } & \multirow{2}{*}{$\mathrm{S}$} \\
\hline & & & & Start & End & Sch & \\
\hline \multirow{2}{*}{1} & \multirow{2}{*}{0} & \multirow{2}{*}{5} & \multirow{2}{*}{$0-1-5$} & 0 & 1 & 0 & \multirow[t]{2}{*}{$0.091,0.083,0.321,0.787$} \\
\hline & & & & 1 & 5 & 0 & \\
\hline \multirow{2}{*}{2} & \multirow{2}{*}{8} & \multirow{2}{*}{4} & \multirow{2}{*}{$8-7-4$} & 8 & 7 & 8 & \multirow[t]{2}{*}{$1.022,0.081,0.091$} \\
\hline & & & & 7 & 4 & 8 & \\
\hline \multirow{2}{*}{3} & \multirow{2}{*}{7} & \multirow{2}{*}{11} & \multirow{2}{*}{$7-9-11$} & 7 & 9 & 7 & \multirow[t]{2}{*}{$0.083,0.321,0.787$} \\
\hline & & & & 9 & 11 & 7 & \\
\hline
\end{tabular}

2. Message forwarding incurs slot to the RN. Slot Range (SR) is defined as the slot of offering the current message of service to the clients. This slot may also include a variable part depending on the current message time left on the mobile device. If the $\mathrm{Q}$ is even small, the user may wait a long time of its depletion and hence leads to a higher SR. Clearly, each $\mathrm{RN}$ has its own valuation of resources. Therefore, their $\mathrm{SR}$ is likely to be different i.e each $\mathrm{RN}$ is free to choose any SR for itself.

3. Assume the Slot Range is SR and the queue length curve fig. 2 is defined in Eq.2. The slot function can be computed as:

$$
\operatorname{profit}(S)=(S-S R) Q
$$

Then, substitution (2) in (3)

$$
\operatorname{profit}(S)=1-S . S m-\frac{S R}{S}+S R . S m \text {. }
$$

4. In our algorithm, we let the RN probe the client(s) to determine the optimal slot.

5. The rerouting may be either locally constructed or fully reconstructed from the source to all nodes.

6. Simple admission control is performed at each node during path construction. A messages is admitted only when the sum of the reservation levels (i.e. $\operatorname{Resv}(1)>$ $\operatorname{Resv}(2)>\operatorname{Resv}(3)>\ldots \ldots . .>\operatorname{Resv}(\mathrm{k}))$ of all messages, including the newly arriving one is less than or equal to the target link utilization.

\section{RESULTS}

In this section we conduct NS-2 simulations to evaluate the different slot mechanisms in a wireless hotspot network. There are three types of nodes in our simulation: base station (BS), relaying nodes (RN), and clients. Fig.(3) shows the topology of experiments, we use experiment $(1-m-n)$ to denote a simulated network scenario where there are one BS, RNs, and clients.

Scheduler Slot Time Scenarios: We consider the network is a scenario classify three different experiment $(\exp 1, . ., \exp 3)$ shown in Table 1. Their differences are: 1) the number of RNs in the network; 2) whether communication exists among the RNs; and 3) whether the clients can reach all

RNs or only a subset of them. Each of these experiment leads to a different slot scheduler time.exp1 depicts a specific waiting slot where the RN scheduler slot time maximize its profit.exp2 is a simple competitive scheduler slot time where a client can choose service from every $\mathrm{RN}$, and the RNs are aware of each other's slot time. In exp3, since the RNs now do not have information about each other's costs, a concurrent scheduler slot time method is used by each $\mathrm{RN}$ to determine its optimal slot. We investigate the scheduler slot time in equilibrium and show that in order to have a stable time, maintaining communication among the RNs is very important

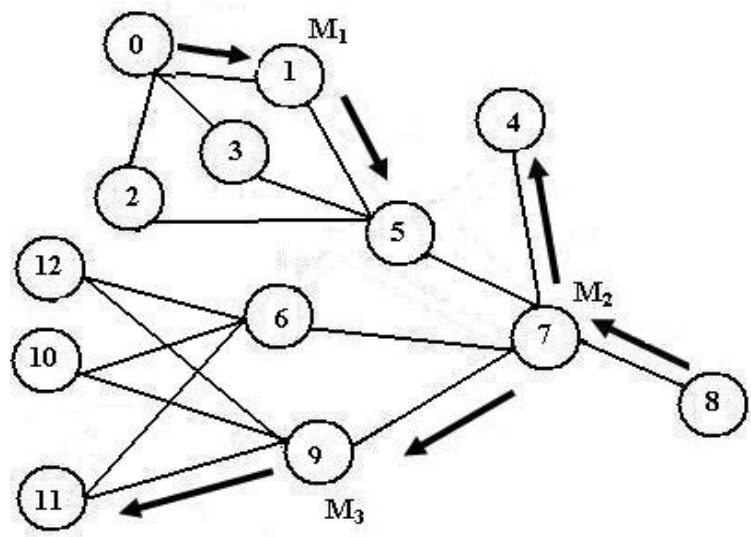

Fig. 3: Topology of experiments

Table 1: Parameters in Experiment

\begin{tabular}{cccc}
\hline Experiment & $\begin{array}{c}\text { Topology of } \\
\text { experiment }\end{array}$ & $\mathrm{S}_{\mathrm{m}}$ of client & $\mathrm{S}$ \\
\hline Exp1(1-1-4) & $8-7-4,5,6,9$ & $0.016,0.028$, & \\
& & & $0.092,0.181$ \\
Exp2(1-3-1) & $0-1,2,3-5$ & 0.008 & 0.083, \\
& & & 0.321, \\
& & $0.009,0.018$, & 1.022, \\
Exp3(1-2-3) & $7-6,9-10,11,12$ & 0.026 & 0.081 \\
\hline
\end{tabular}


Analytical Versus Simulation Results: We verify our analysis via simulation. Three experiment are randomly generated in the same network as in Section 4: three guaranteed experiment and two best effort flows. The network topology information are shown in Fig. 3. The comparison of the analytical result with the simulation result is shown in Table 1. We see that our analytical model provides very good estimation for the performance of scheduler slot time. In an ad hoc network, a transmitting node, say node, will interfere with another node, say, if either condition holds. Node is within the transmission range of node and is the receiver of another message. We consider the two-state error model. Assume there are message in the ad hoc network. The average number of slot time for a message is, where is the measurement scheduler time, and is the steady-state probability of the good state. Let indicate the set of messages passing through node, and represents the set of messages interference range. For each forwarding messages passing through node, its bandwidth share is, where is the number of messages managed by node. Furthermore, the information of messages and the corresponding interfering nodes is shown in Table 2.

\section{CONCLUSION}

In this paper we focus on the message forwarding incentive problem in a multihop wireless hotspot network. We model the system as a Queue where the timing for message forwarding is determined by queue lenght. We classify the scenario into three parts, and propose different time solutions for each of them. In particular, we design a SSM algorithm for a Queue lenght, and introduce a SR-like marginal Slot range for a Queue with multiple relaying nodes which guarantees truthful reporting of marginal slots. We further extend the network scenarios to cover the situation where a client can only reach a subset of the relaying nodes, and another situation where the relaying nodes do not have communication among them. Our simulation results show that the slot mechanisms are able to guide the network into an equilibrium state quickly, and that keeping communication among the relaying nodes is important to maintain a stable network. Therefore, the base station's helping to maintain communications among the set of relaying nodes is beneficial for the whole network.

In the future, we will investigate fair scheduling with QoS metrics such as delay or delay jitter for ad hoc networks.

\section{REFERENCES}

1. H. Luo and S. Lu, 2000. A topology-independent fair queueing model in ad hoc wireless networks, in Proc. IEEE Int. Conf. Network Protocol.
2. H. Luo, P. Medvedev, J. Cheng, S. Lu 2001 .A self-coordinating approach to distributed fair queueing in ad hoc wireless networks, in Proc. IEEE INFOCOM , pp : 1370-1379.

3. H. L. Chao and W. Liao, 2002. Fair scheduling with QoS support in ad hoc networks, in Proc. IEEE Conf. Local Computer Networks.

4. P. Goyal, H. M. Vin, and H. Cheng,1997.Start-time fair queueing: A scheduling algorithm for integrated services packet switching networks," IEEE/ACM Trans. Networking, vol. 5, no. 5.

5. S. Lee and M. Gerla,2001. DLAR: Dynamic: load aware routing in ad hoc networks, Proc. ICC 2001, Helsinki, pp:3206-3210 .

6. M. R. Pearlman, Z. J. Haas, P. Sholander, S. S Tabrizi,2000.On the Impact of Alternate Path Routing for Load Balancing in Mobile Ad Hoc Networks, Proceedings of the ACM MobiH6c,pp: 3-10.

7. P. Pham and S. Perreau, 2003. Performance Analysis of Reactive Shortest Path and Multi-Path Routing Mechanism with Load Balance. In Proc. IEEE INEOCOM, IEEE, pp:251-259.

8. Y. Wang, H. Lin, and S. Chang, 2004. Interferingaware QoS Multipath Routing for Ad Hoc Wireless Network. Proceedings of the 18th Interntional Conference on Advanced Information Networking and Application (AINA'04), pp 29-34.

9. A. Balachandran, G. Voelker, and P. Bah ,2003. Wireless hotspots: Current challenges and future directions. In Proc. The First ACM Intl. Workshop on Wireless Mobile Applications and Services on WLAN Hotspots (WMASH'03), San Deigo, California, U.S.A.

10. F. Fitzek, D. Angelini, G. Mazzini, and M. Zorzi.2003. Design and performance of an enhanced IEEE 802.11 MAC protocol for multihop coverage extension. IEEE Wireless Communications, pp:30-39.

11. L. Kleinrock, 1976. Queueing Systems, vol. 2, chap. 3, pp: 119-126, John Weiley and Sons.

12. V. Bhargavan S. Lu and R. Srikant,1999. Fair scheduling in wire- less packet networks, IEEELACM Transactions on Networking, vol. 7, no. 4, pp: 473-489.

13. A. Ephremides and T. Truong, 1988. Distributed algorithm for efficient and interference-free broadcasting in radio networks, in Proceedings of IEEE INFOCOM, pp:1119-1124.

14. G Lu, D Belis, Dr. G Manson,2005. A new approach for generating mobility scenarios towards environment aware simulation in MANET, in Proceeding of PREP2005, UK.

15. L. Huang and T.-H. Lai,2002. On the scalability of IEEE 802.11 ad hoc networks, in Proc. Mobi Hoc, pp:173-182

16. T.-H. Lai and D. Zhou,2003. Efficient and scalable IEEE 802.11 ad-hoc mode timing synchronization function, in Proc. 17th IEEE Int. Conf. Advanced Information Networking Applicat. 